\title{
Clinical, Psychopathological, Physical, and Sleep Evolution in Adolescents with Restrictive Anorexia Nervosa Participating in a Day Hospital Program
}

\author{
Sofía M Martínez-Sánchez ${ }^{1 凶}$, Tomás E Martínez-García², and Diego Munguía-Izquierdo ${ }^{3,4}$ \\ 'Department of Sports and Computer Science, Section of Physical Education and Sports, Faculty of Sports Sciences, Universidad Pablo de Olavide, \\ Seville, Spain \\ 2Department of Internal Medicine, Juan Ramón Jiménez Hospital, Huelva, Spain \\ ${ }^{3}$ Physical Performance Sports Research Center, Department of Sports and Computer Science, Section of Physical Education and Sports, \\ Faculty of Sports Sciences, Universidad Pablo de Olavide, Seville, Spain \\ ${ }^{4}$ Biomedical Research Networking Center on Frailty and Healthy Aging, Madrid, Spain
}

Objective To analyze the clinical, psychopathological, physical, and sleep-related evolution of adolescents with restricting-type of anorexia nervosa (AN-R) after 10 weeks of a daytime hospital program.

Methods Body composition, physical activity and sleep were measured objectively before and after 10 weeks of treatment. In addition, psychopathology and body image disturbances were measured with a self-report questionnaire.

Results Fourteen female adolescents with AN-R (14.3 \pm 1.6 years old) participated in the study. A significant increase was found in eight of the ten variables for body composition $(\mathrm{p}<0.05)$. There were no significant changes in psychopathology, body image disturbances or physical activity. Concerning sleep, a significant, moderately standardized and substantial increase in night latency was found $(\mathrm{p}=0.002)$, and there was a significant, small standardized and substantial decrease in night efficiency $(\mathrm{p}=0.035)$.

Conclusion After 10 weeks of follow-up with adolescent patients with AN-R who attended a day hospital program, there was a positive evolution of body composition. However, with regard to sleep patterns, there was a worsening of latency and night efficiency. Therefore, sleep care should be addressed in acute treatment programs for adolescents with AN-R. Psychiatry Investig 2020;17(4):366-373

Key Words Restrictive anorexia nervosa, Adolescents, Sleep, Physical activity, Psychopathology.

\section{INTRODUCTION}

Anorexia nervosa (AN) is a severe psychiatric illness that is prone to pain chronification and is associated with an increased mortality risk. ${ }^{1}$ AN occurs mainly during adolescence, with prevalence rates of approximately $1.7 \%$ in adolescents. ${ }^{2}$ A low body weight, a fear of gaining weight and an alteration of body image are characteristics of AN. The restrictive subtype of $\mathrm{AN}$ (AN-R) occurs when weight loss is primarily due to diet, fasting and/or excessive exercise and, unlike the $\mathrm{AN}$

Received: January 20, 2020 Revised: February 9, 2020

Accepted: February 15, 2020

$\square$ Correspondence: Sofía M Martínez-Sánchez, MSc

Department of Sports and Computer Science, Section of Physical Education and Sports, Faculty of Sports Sciences, Universidad Pablo de Olavide, Carretera de Utrera Km. 1, s/n, Seville 41013, Spain

Tel: +34-954977589, E-mail: sofiams91@gmail.com

(c) This is an Open Access article distributed under the terms of the Creative Commons Attribution Non-Commercial License (https://creativecommons.org/licenses/bync/4.0) which permits unrestricted non-commercial use, distribution, and reproduction in any medium, provided the original work is properly cited. binge-purge subtype, includes more discrete and extreme instances of reducing intake, as well as more chronic reduction of energy. ${ }^{3}$ Differences in body composition and psychopathology have been demonstrated between the subtypes of AN. ${ }^{4}$ Therefore, it is necessary to differentiate the evolution of the illness between the AN subtypes to contribute to a better understanding of the symptomatology and course of AN and to find more specific treatment approaches.

Although a recent systematic review highlighted the lack of consensus on treatment protocols with respect to refeeding practices, ${ }^{5}$ the importance of rapid refeeding strategies for body weight restoration in this population is clear. Restoring optimal weight, especially in AN-R, is the key to avoiding serious physical complications in the bone mineral density, the musculoskeletal system and linear growth. ${ }^{6}$ Therefore, monitoring these systems during the refeeding process is essential among these patients. There is currently no superior treatment setting for AN (outpatient, inpatient, reduced length of inpatient fol- 
lowed by outpatient, or partial hospital care). ${ }^{7}$ However, programs based on cognitive behavioral treatment, together with family support, seem to be effective and have been recommended in the treatment of adolescents with AN. ${ }^{8}$

In addition to inpatient care, additional goals must be completed to facilitate a movement toward full recovery. ${ }^{9}$ The available studies examining the changes in the psychopathology of adolescents with AN in hospital environments show contradictory results. Some studies found an improvement in general psychopathology, ${ }^{10,11}$ while others did not find changes despite weight recovery. ${ }^{12}$ Likewise, research on the efficacy and impact of interventions for AN should include evaluation of body image disturbance, in addition to changes in weight and medical stability. ${ }^{13}$

An increase in physical activity (PA) can be considered a significant factor for the onset and maintenance of the illness. ${ }^{3}$ Since these patients are actively trying to modify the shape of their body, PA is only one of the ways to obtain the "desired ideal thinness." In addition, these patients may rely on PA as a key coping strategy in the face of negative affect of the illness. ${ }^{14}$ However, few studies have objectively evaluated the changes in PA during nutritional recovery in patients with $\mathrm{AN}$, and different results have been obtained. ${ }^{15}$ As a consequence, a better understanding of PA in this population is justified during weight restoration efforts to improve outcomes for patients with AN-R.

Sleep disorders are highly correlated with AN and that there are multiple areas of sleep that AN effects compared to a healthy population. ${ }^{16}$ In fact, sleep disorders in patients with AN have been considered an important clinical marker. ${ }^{17}$ To date, the studies that have examined sleep disorders among patients with AN are mainly using polysomnographic monitoring. ${ }^{18}$ However, studies are needed to evaluate sleep in a natural environment over a long period, and for this, actigraphs are a useful method for the assessment of sleep. ${ }^{19}$

Therefore, the objective of the present study was to analyze the clinical, psychopathological, physical, and sleep-related evolution of adolescents with AN-R after 10 weeks of a daytime hospital program. We hypothesized that after the hospital program it would improve the parameters evaluated in adolescents with AN-R.

\section{METHODS}

\section{Participants}

This study was approved by the Research Ethics Committee of the University Hospital Complex of Huelva (PI 005/16) and registered in www.clinicaltrials.gov (Identifier: NCT03667183), and it followed the guidelines of the Declaration of Helsinki, last-modified in 2013. All female participants between 10 and
17 years old with restricting-type AN ( $n=23)$, who were diagnosed according to the clinical criteria of the fifth edition of the Diagnostic and Statistical Manual of Mental Disorders $(\mathrm{DSM}-5)^{3}$ and who were included in the day hospital program (DHP) of the Treatment Program for Eating Disorders (TPED) were recruited from the Children's Mental Health Unit of the Vázquez Díaz Hospital (Huelva, Spain). Fourteen subjects agreed to participate, and they and their parents signed written informed forms. The inclusion criteria were as follows: 1) clinical diagnosis of restricting-type $\mathrm{AN}$ in the aforementioned hospital; 2) aged from 10 to 17 years old; 3) the medical team indicated that patients were under analytical control to participate; and 4) written informed consent of the patients and their legal guardians was provided. The exclusion criteria were 1) having other mental health diagnoses and 2) consumption of narcotic drugs. All patients were assessed by the same researcher to reduce interexaminer error.

\section{Treatment description}

The DHP of the TPED is a program that has been held at the Child-Mental Health Unit of the Vázquez Díaz Hospital (Huelva, Spain) since 2001. The multidisciplinary team is composed of eleven specialized eating disorders (EDs) health professionals (one psychiatrist, one endocrinologist, three nurses, four nursing assistants, one occupational therapist and one social worker) together with a professor and an administrative officer. The main objective of the program is to reduce the problematic food-related symptoms, such as restriction, binging and purging, of children who need intensive treatment without being totally hospitalized. The DHP characteristics of TPED are described in the Supplementary material.

\section{Procedure}

The study consisted of four visits to the hospital. In the first visit (baseline), anthropometric data and body composition data were examined, and patients were monitored for energy expenditure for 9 days. The day where the portable monitor was removed, they also completed different questionnaires. The procedure was repeated 10 weeks later. The delay between admission to the hospital and baseline assessment averaged 48.7 ( \pm 11.6$)$ days. All measurements and questionnaires were collected and supervised by qualified personnel.

\section{Clinical measures}

InBody 770 (Inbody Co., LTD, Seoul, Korea) was used to measure body composition through bioelectrical impedance analysis (BIA). Multifrequency and octopolar bioelectrical impedance, such as InBody 770, has been shown to be reliable and precise in patients with $\mathrm{AN} .{ }^{20}$ This body composition analyzer has already been validated in children and adolescents. ${ }^{21}$ 
Height was measured barefoot and in underwear to the nearest $0.1 \mathrm{~cm}$ following standard procedures using a balance with an incorporated stadiometer (Detecto 439; Detecto, USA). Body mass index (BMI) was calculated as body mass (kg) divided by height $(\mathrm{m})$ squared.

\section{Psychopathology of AN}

\section{Psychiatric symptoms}

The self-report questionnaire Eating Disorder Inventory-3 (EDI-3) was used to analyze psychological traits and key symptoms of the development and maintenance of EDs, including AN. ${ }^{22}$ It is composed of 91 items with a choice of 6 answers each and organized into 12 main scales: three scales specific to EDs and nine general psychological scales that are highly relevant but not specific to EDs. The EDI- 3 also provides 6 composite scores: a specific one for EDs and five indices of integrative psychological constructs. We used the Spanish adaptation of the EDI- $3,{ }^{22}$ which has high levels of internal consistency in all the diagnostic groups (Cronbach's alpha from 0.85 to 0.95$)$.

\section{Body image disturbance}

The Contour Drawing Rating Scale (CDRS) was used because it is one of the easy-to-administer and most popular figure drawing scales for the evaluation of body image disturbance. $^{23,24}$ This self-administered scale is composed of nine female figures viewed from the front with relatively fine increasing graduations of BMI. The person is asked to indicate which figure represents their current body shape (perceived body shape) and what figure they would like to have (ideal body shape). The discrepancy between these two classifications represents a measure of body dissatisfaction. A score of 0 was interpreted as satisfaction with body image, and a score different than 0 was interpreted as body dissatisfaction (Satisfied $=0$; Dissatisfied $=1$ ). ${ }^{25}$ The use of the absolute value of the discrepancy between the current and desired body shape was used in the analyses, as it has also been used previously. ${ }^{26}$ The validity and reliability test-retest of the CDRS have been satisfactorily analyzed in adolescents (Cronbach's alpha from 0.77 to 0.84$),{ }^{24}$ and it has been widely used in clinical and healthy populations. $^{27}$

\section{Physical activity and sleep}

Free-living activity behaviors were objectively measured using the multisensor monitor Sensewear Mini Armband (SWM) (BodyMedia Inc., Pittsburgh, PA, USA), previously used in adult patients with $\mathrm{AN},{ }^{28}$ for a 24-hours, 9-days period, which included five weekdays and two weekend days. Sleep time and sedentary ( $\leq 1.5 \mathrm{METs})$, light (1.6-2.9 METs), moderate (3-6 METs) and vigorous ( $>6$ METs) physical activity were recorded on a minute-by-minute basis. Sleep time variables were night sleep duration (time from sleep onset to offset), sleep latency (time from bed time to sleep start), night perturbations (number and time of perturbations after sleep onset) and sleep efficiency (percentage of time spent asleep from sleep onset to offset). Participants were told to remove the monitor only for water-based activities. Only participants who carried the monitor for at least $95 \%$ of the entire day $(1,368$ minutes) were included in the study. To minimize immediate reactivity to the monitor that may have altered their habitual lifestyle, we removed from the analysis the first and the last day of monitoring data.

\section{Statistical analyses}

All statistical tests were performed using SPSS (SPSS, IBM Corp. Released 2016. IBM SPSS Statistics for Windows, Version 24.0. IBM Corp., Armonk, NY, USA). Significance was set at $\mathrm{p}<0.05$. Due to the size of the sample, we assumed a nonnormal distribution; therefore, the Wilcoxon test was used to evaluate significant differences between baseline assessment and after 10 weeks, and when there was significance, the $95 \%$ confidence intervals (CI) for the differences were calculated. Data were also assessed for practical/clinical meaningfulness using an approach based on the magnitudes the changes observed. Cohen's d statistic determined the effect size of the standardized differences in the selected variables, and Hopkins' scale and a customized spreadsheet were used to determine the magnitude of the effect size. ${ }^{29,30}$ A practically worthwhile difference was assumed when the difference score of the between subject standard deviation was at least 0.2 . Threshold values for Cohen's effect size were trivial (0.0-0.19), small (0.20-0.59), moderate (0.60-1.19), large (1.20-1.99), and very large ( $\geq 2.00)$. Quantitative chances of positive/trivial/negative difference were assessed qualitatively as follows: $<25 \%$ unclear, $25 \%$ to $75 \%$ possibly, $>75 \%$ likely, $>95 \%$ very likely, and $>99.5 \%$ almost certainly. A substantial difference was set at $>75 \%$.

\section{RESULTS}

The average age of the 14 participants was $14.3( \pm 1.6)$ years. The results of the clinical evolution of the patients after 10 weeks of the intervention program are shown in Table 1. A significant, small standardized and substantial increase was found in weight ( $\mathrm{p}=0.009,95 \% \mathrm{CI}$ : -3.57 to -0.75 ) and body mass index ( $p=0.019,95 \%$ CI: -1.27 to -0.16$)$. Significant and small standardized increases were found in total body water ( $\mathrm{p}=0.014,95 \% \mathrm{CI}:-1.29$ to -0.21 ), body fat mass ( $\mathrm{p}=0.028,95 \%$ CI: -2.26 to -0.04 ), soft lean mass ( $\mathrm{p}=0.011,95 \% \mathrm{CI}:-1.65$ to 
-0.32), fat-free mass ( $\mathrm{p}=0.014,95 \% \mathrm{CI}-1.74$ to -0.29$)$ and skeletal muscle mass ( $\mathrm{p}=0.010,95 \% \mathrm{CI}:-1.08$ to -0.22 ). Moreover, there was a significant increase in height $(\mathrm{p}=0.003,95 \% \mathrm{CI}$ :
-1.27 to -0.37$)$ and a small standardized increase in percent body fat.

The evolution of psychopathology is shown in Table 2. In

Table 1. The evolution of body composition between baseline assessment and after 10 weeks

\begin{tabular}{|c|c|c|c|c|c|}
\hline & Baseline $(\mathrm{N}=14)$ & After 10 weeks $(\mathrm{N}=14)$ & Standardized differences (90\% CL) & \multicolumn{2}{|c|}{ Qualitative assessment $^{\dagger}$} \\
\hline Weight (kg) & $45.2(7.8)$ & $47.4(6.5)^{*}$ & $0.28(0.14)$ & Likely positive & $82 / 18 / 0$ \\
\hline Height $(\mathrm{cm})$ & $158.9(7.6)$ & $159.8(7.3)^{*}$ & $0.10(0.05)$ & Almost certainly trivial & $0 / 100 / 0$ \\
\hline Total body water (L) & $24.9(3.4)$ & $25.6(2.9)^{*}$ & $0.21(0.12)$ & Possibly positive & $54 / 46 / 0$ \\
\hline Body fat mass (kg) & $11.3(4.4)$ & $12.4(4.3)^{*}$ & $0.26(0.18)$ & Possibly positive & $70 / 30 / 0$ \\
\hline Soft lean mass (kg) & $31.9(4.4)$ & $32.9(3.7)^{*}$ & $0.21(0.11)$ & Possibly positive & $56 / 44 / 0$ \\
\hline Fat free mass $(\mathrm{kg})$ & $34.0(4.7)$ & $35.0(3.9)^{*}$ & $0.20(0.12)$ & Possibly positive & $53 / 47 / 0$ \\
\hline Skeletal muscle mass (kg) & $18.0(2.8)$ & $18.6(2.4)^{*}$ & $0.22(0.12)$ & Possibly positive & $60 / 40 / 0$ \\
\hline Body mass index $\left(\mathrm{kg} / \mathrm{m}^{2}\right)$ & $17.9(2.6)$ & $18.6(2.1)^{*}$ & $0.27(0.17)$ & Likely positive & $76 / 24 / 0$ \\
\hline Percent body fat (\%) & $24.3(6.9)$ & $25.7(6.6)$ & $0.20(0.20)$ & Possibly positive & $50 / 50 / 0$ \\
\hline Bone mineral content $(\mathrm{kg})$ & $2.1(0.3)$ & $2.1(0.2)$ & $0.13(0.13)$ & Possibly trivial & $18 / 82 / 0$ \\
\hline
\end{tabular}

Data are shown as the mean (SD), unless otherwise indicated. Body composition differences between baseline assessment and after 10 weeks were analyzed by the Wilcoxon test. ${ }^{*} \mathrm{p}<0.05$, ${ }^{\dagger}$ a substantial difference was set at $>75 \%$. CL: confidence level

Table 2. Psychopathological evolution between baseline assessment and after 10 weeks

\begin{tabular}{|c|c|c|c|c|c|}
\hline \multirow[b]{2}{*}{ Eating disorder inventory- $3^{\dagger}$} & \multirow[t]{2}{*}{ Baseline $(\mathrm{N}=14)$} & \multirow[t]{2}{*}{ After 10 weeks $(\mathrm{N}=14)$} & \multirow[t]{2}{*}{$\begin{array}{c}\text { Standardized } \\
\text { differences }(90 \% \mathrm{CL})\end{array}$} & \multicolumn{2}{|c|}{ Qualitative assessment* } \\
\hline & & & & & \\
\hline Drive for thinness & $42.4(7.4)$ & $40.9(7.2)$ & $-0.21(0.24)$ & Possibly negative & $1 / 48 / 52$ \\
\hline Bulimia & $45.9(4.8)$ & $44.7(2.7)$ & $-0.21(0.32)$ & Possibly negative & $2 / 47 / 51$ \\
\hline Body dissatisfaction & $42.3(7.7)$ & $42.7(7.0)$ & $0.07(0.27)$ & Possibly trivial & $21 / 74 / 5$ \\
\hline Eating disorder risk composite & $41.7(6.7)$ & $40.8(6.4)$ & $-0.13(0.23)$ & Possibly trivial & $1 / 69 / 29$ \\
\hline Low self-esteem & $44.4(7.9)$ & $44.0(8.2)$ & $-0.06(0.27)$ & Unclear & $6 / 77 / 18$ \\
\hline Personal alienation & $44.4(8.3)$ & $43.9(8.3)$ & $-0.05(0.31)$ & Unclear & $9 / 70 / 21$ \\
\hline Interpersonal insecurity & $46.6(9.9)$ & $46.6(11.1)$ & $-0.02(0.34)$ & Unclear & $14 / 67 / 18$ \\
\hline Interpersonal alienation & $46.4(9.4)$ & $47.8(9.4)$ & $0.14(0.31)$ & Possibly trivial & $38 / 59 / 4$ \\
\hline Interoceptive deficits & $44.7(8.1)$ & $42.6(8.5)$ & $-0.26(0.39)$ & Possibly negative & $3 / 37 / 60$ \\
\hline Emotional dysregulation & $46.9(7.6)$ & $46.5(9.8)$ & $-0.09(0.33)$ & Unclear & $7 / 65 / 28$ \\
\hline Perfectionism & $46.7(5.5)$ & $48.1(9.4)$ & $0.15(0.60)$ & Unclear & $45 / 40 / 16$ \\
\hline Asceticism & $43.4(7.0)$ & $42.1(6.3)$ & $-0.16(0.47)$ & Unclear & $10 / 46 / 44$ \\
\hline Maturity fears & $52.3(5.9)$ & $55.3(8.5)$ & $0.41(0.44)$ & Likely positive & $79 / 19 / 1$ \\
\hline Ineffectiveness & $44.1(7.1)$ & $43.6(8.1)$ & $-0.07(0.30)$ & Unclear & $7 / 71 / 22$ \\
\hline Interpersonal problems & $46.2(9.4)$ & $47.0(10.8)$ & $0.06(0.27)$ & Unclear & $19 / 75 / 6$ \\
\hline Affective problems & $45.3(7.0)$ & $43.9(7.4)$ & $-0.19(0.31)$ & Possibly negative & $2 / 49 / 49$ \\
\hline Overcontrol & $44.2(5.9)$ & $44.3(7.8)$ & $-0.03(0.54)$ & Unclear & $23 / 47 / 29$ \\
\hline General psychological maladjustment & $44.4(6.1)$ & $44.6(8.6)$ & $-0.04(0.33)$ & Unclear & $12 / 69 / 20$ \\
\hline \multicolumn{6}{|l|}{ Contour drawing rating scale } \\
\hline Perceived body shape & $4.6(1.3)$ & $4.9(1.2)$ & $0.26(0.33)$ & Possibly positive & $63 / 36 / 1$ \\
\hline Ideal body shape & $4.4(1.2)$ & $4.6(1.2)$ & $0.14(0.20)$ & Possibly trivial & $32 / 68 / 1$ \\
\hline Body dissatisfaction ${ }^{\ddagger}$ & $0.7(0.5)$ & $0.6(0.5)$ & $-0.29(0.63)$ & Unclear & $10 / 31 / 59$ \\
\hline
\end{tabular}

Data are shown as the mean (SD), unless otherwise indicated. Psychological evolution differences between baseline assessment and after 10 weeks were analyzed by the Wilcoxon test. *a substantial difference was set at $>75 \%$, ${ }^{\dagger}$ all scales and composite scores of Eating Disorder Inventory-3 are shown in T-scores, ${ }^{\dagger}$ the greater values indicate greater body dissatisfaction. CL: confidence level 
relation to EDI-3, small standardized and substantial increases were found in maturity fears, and small standardized decreases were obtained in drive for thinness, bulimia and interoceptive deficits. Regarding CDRS, there was a small standardized increase in perceived body shape and a decrease in body dissatisfaction.

Changes in physical activity and sleep are shown in Table 3. In relation to physical activity, there was a small standardized decrease in daily average METs and an increase in sedentary activity. With regard to sleep, a significant, moderately standardized and substantial increase in sleep latency was found ( $\mathrm{p}=0.002$, 95\% CI: -16.00 to -4.10 ), and a significant, small standardized and substantial decrease in sleep efficiency was found ( $p=0.035,95 \%$ CI: -0.04 to 5.37 ). In addition, small standardized and substantial increases were found in night perturbations, both in number and in duration.

\section{DISCUSSION}

This study analyzed the clinical, psychopathological, physi$\mathrm{cal}$, and sleep evolution of female adolescents with restrictingtype AN admitted to a day hospital program for 10 weeks. The main finding, in addition to the increase in body mass, was the increase in sleep latency and, consequently, the decrease in sleep efficiency.

A major finding of the current study was that the measured sleep objectively worsened after refeeding in adolescents with
AN-R. We found an increase in nocturnal latency and the number and duration of night perturbations, which is because there was a decrease in sleep efficiency. It has been reported that there is an objectively measured improvement in sleep after the restoration of weight in patients with $\mathrm{AN}^{31}$ and that sleep disorders are related to the malnutrition observed in these patients, and, after increasing weight, sleep disorders improve. ${ }^{16,31}$ However, Pieters et al. ${ }^{18}$ did not observe changes in the objective variables of sleep after 20 weeks of treatment in adolescents with $\mathrm{AN}$, and Lehmann et al. ${ }^{32}$ found that the duration of sleep was inversely associated with an increase in BMI. Anxiety triggered by the increase in weight and the pressure of monitoring and control of physical activity by hospital staff and parents could be one of the explanations for the increase in sleep disorders in this clinical population after 10 weeks of refeeding. ${ }^{17}$ Therefore, it is crucial to address sleep care in acute treatment protocols in adolescents with AN-R to potentiate the treatment efficacy for the main clinical disorder, as evidenced by other psychiatric conditions. ${ }^{16}$

In our study we found a significant increase in eight of the ten variables of body composition, in agreement with other studies of refeeding in this population. ${ }^{33,34}$ Contrary to our results, other study reported a decrease in total body water after weight recovery in adults with $\mathrm{AN}^{34}$ This discrepancy could be due to the comparison of adults with adolescents, since an increase in total body water was also reported after refeeding in adolescents with $\mathrm{AN}$, and this increase was due to the in-

Table 3. Changes in physical activity and sleep between baseline assessment and after 10 weeks

\begin{tabular}{|c|c|c|c|c|c|}
\hline & Baseline $(\mathrm{N}=14)$ & $\begin{array}{l}\text { After } 10 \text { weeks } \\
\qquad(\mathrm{N}=14)\end{array}$ & $\begin{array}{c}\text { Standardized } \\
\text { differences }(90 \% \mathrm{CL})\end{array}$ & \multicolumn{2}{|c|}{ Qualitative assessment ${ }^{\dagger}$} \\
\hline Energy expenditure (kcal/day) & $1876.9(248.1)$ & $1890.7(244.2)$ & $0.06(0.30)$ & Unclear & $20 / 72 / 7$ \\
\hline Active energy expenditure (kcal/day) & $967.6(278.8)$ & $933.0(339.3)$ & $-0.14(0.54)$ & Unclear & $14 / 43 / 43$ \\
\hline Steps (number/day) & $7,069(3,036)$ & $7,137(3,372)$ & $0.04(0.31)$ & Unclear & $19 / 72 / 9$ \\
\hline Average $\mathrm{METs}^{\ddagger}$ & $1.8(0.2)$ & $1.7(0.3)$ & $-0.35(0.39)$ & Possibly negative & $1 / 25 / 74$ \\
\hline Sedentary activity $<1.5$ METs (min/day) & $783(337)$ & $857(268)$ & $0.26(0.35)$ & Possibly positive & $61 / 37 / 2$ \\
\hline Light activity 1.6-2.9 METs (min/day) & $545(339)$ & $472(254)$ & $-0.17(0.34)$ & Possibly trivial & $4 / 53 / 43$ \\
\hline Moderate activity 3-6 METs (min/day) & $75(40)$ & $68(47)$ & $-0.11(0.37)$ & Unclear & $8 / 58 / 34$ \\
\hline Vigorous activity $>6$ METs (min/day) & $19(19)$ & $22(27)$ & $0.06(0.28)$ & Unclear & $20 / 74 / 6$ \\
\hline \multicolumn{6}{|l|}{ Sleep } \\
\hline Night sleep duration (min/day) & $491(59)$ & $493(41)$ & $0.06(0.53)$ & Unclear & $32 / 47 / 20$ \\
\hline Sleep latency (min/day) & $11(6)$ & $21(11)^{*}$ & $0.97(0.39)$ & Almost certainly positive & $100 / 0 / 0$ \\
\hline Night perturbations (number/day) & $10(5)$ & $13(4)$ & $0.54(0.52)$ & Likely positive & $86 / 13 / 1$ \\
\hline Night perturbations (min/day) & $54(33)$ & $66(21)$ & $0.47(0.37)$ & Likely positive & $89 / 11 / 0$ \\
\hline Sleep efficiency (\%) & $90.6(5.6)$ & $88.0(4.6)^{*}$ & $-0.43(0.38)$ & Likely negative & $1 / 15 / 84$ \\
\hline
\end{tabular}

Data are shown as the mean (SD), unless otherwise indicated. Changes in physical activity and sleep between baseline assessment and after 10 weeks were analyzed by the Wilcoxon test. ${ }^{*} \mathrm{p}<0.05,{ }^{\dagger}$ a substantial difference was set at $>75 \%$, ${ }_{\text {daily }}$ average. CL: confidence level, METs: metabolic equivalents 
crease in intracellular water. ${ }^{35}$ Unlike our results, there was a significant increase in bone mineral content in a study of adolescents with $\mathrm{AN}$; however, no changes were found in lean tissue mass after 7 months of weight gain. ${ }^{33}$ It is likely that this result is due to the study lengths, since no changes were found in whole body bone mineral density after a 12 -week intensive nutrition therapy program with AN patients. ${ }^{36}$

We did not find any significant change in the psychopathology of the anorexia nervosa or in the evaluation of body dissatisfaction. These results agree with other previous studies in which no significant improvement was obtained after weight recovery in adolescents with AN. ${ }^{12,37}$ However, Iniesta Sepúlveda et al. ${ }^{10}$ observed a decrease in core pathological features, while Hatch et al. ${ }^{11}$ obtained improvements in only two of the five psychopathological variables. Several studies have reported the existing gap between physical recovery and psychological change since they did not find significant associations between them, ${ }^{12,34}$ which could explain the high rate of relapse of AN after discharge from inpatient units. One of the reasons that can explain this gap in our results may be the short evaluation period between the two assessments of our study. On the other hand, body image disturbance does not seem to change after hospitalization in these patients. ${ }^{12,38}$ Kodama et al. ${ }^{38}$ found, through functional magnetic resonance imaging, that there is an alteration of body image even after weight recovery in patients with AN. In our patients, we observed a small increase in perceived body image and a decrease in body dissatisfaction. Similar findings were obtained in a previous study in which ideal body image did not change after 12 weeks of nutritional recovery, though patients did obtain an increase in perceived body image. ${ }^{39}$ It has been reported that younger adolescents with AN perceive greater coercion than older adolescents, ${ }^{37}$ and those who have a lower weight experience less anxiety with body image than those with recovered weight. ${ }^{40}$ Although nutritional restoration is key in the treatment of these patients, the increase in the amount of food increases anxiety and resistance to refeeding; ${ }^{41}$ therefore, these aspects should be treated with greater emphasis especially in this clinical population in treatment programs.

Objective assessment of physical activity in adolescents with AN-R using an activity monitor revealed that there were no changes in activity after 10 weeks of refeeding, except for a small decrease in average METs and a small increase in sedentary activity. This finding contrasts with other study in which an increase in energy expenditure was found after weight recovery in adults. ${ }^{42}$ However, it seems that this increase is not directly related to the severity of the pathology nor does it seem to cause weight loss or relapse during follow-up. ${ }^{42}$ In a study with adolescents with AN, it was reported that physical activity tends to normalize during treatment and remains stable one year after follow-up. ${ }^{15}$ A possible explanation for not finding changes in physical activity in our study could be due to the efforts of the clinical staff not to increase the activities in the patients, since it could compromise the nutritional recovery, which is the priority, in this clinical population.

The main strength of this study is that it is one of the very few to investigate several days of sleep and physical activity (7 full days) objectively in female adolescents with AN-R before and after weight gain. In addition, our sample was homogenous and comprised drug-free, restricting-type AN patients without comorbid depression or other mental health diagnoses. Because of rapid maturational changes, sleep characteristics in young adolescents should be interpreted with great caution. A confounding variable could have been the mean time between admission and baseline assessment. During this period, patients were already in a stage of active refeeding, and this was necessary to participate in the study safely under medical supervision. In addition, if one studies patients shortly after admission, the sleep quality may be influenced by the stress of being admitted to a hospital and/or the adaptation to a new environment.

There are some limitations to be considered regarding our study. First, it is a single-arm study, and therefore, no control group was used for comparison, resulting in time as the independent variable. Second, there are known limitations of all nonprobability samples, including the unknown levels of sampling errors and their lack of representativeness. Third, the small sample in this study may be due to the low prevalence of this illness, and it is difficult to enroll a large number of affected adolescents for ten weeks. Future research should include a randomized controlled design and a larger sample of adolescents to thoroughly validate our results.

In conclusion, after 10 weeks of follow-up in adolescent patients with AN-R who participated in a day hospital program, there was a positive evolution of body composition. No changes were observed in the patients' general psychopathology. However, regarding sleep patterns, there was a worsening of sleep latency and efficiency, so sleep care should be addressed in acute treatment programs for adolescents with AN-R.

\section{Supplementary Materials}

The online-only Data Supplement is available with this article at https://doi.org/10.30773/pi.2020.0016.

\section{Acknowledgments}

This study was funded by Research Group CTS-948 of University Pablo of Olavide (Seville), this funding included material and equipment for the research. There was no external financial support. We sincerely thank all patients for their participation in our study. We gratefully acknowledge the support of the workers of the Child-Mental Health Unit of the Vázquez Díaz Hospital. Special thanks to Lourdes Hernández and Pilar Rodríguez for their assistance with the data collection. 


\section{Conflicts of Interest}

The authors have no potential conflicts of interest to disclose.

\section{Author Contributions}

Conceptualization: all authors. Data curation: all authors. Formal analysis: all authors. Funding acquisition: Tomás E Martínez-García, Diego Munguía-Izquierdo. Investigation: all authors. Methodology: all authors. Project administration: Sofía M Martínez-Sánchez. Resources: Sofía M Martínez-Sánchez, Diego Munguía-Izquierdo. Software: Sofía M MartínezSánchez, Diego Munguía-Izquierdo. Supervision: Tomás E Martínez-García, Diego Munguía-Izquierdo. Validation: all authors. Visualization: all authors. Writing_original draft: Sofía M Martínez-Sánchez. Writing—review \& editing: all authors.

\section{ORCID iDs}

Sofía M Martínez-Sánchez

Tomás E Martínez-García

Diego Munguía-Izquierdo

https://orcid.org/0000-0003-1722-2470

https://orcid.org/0000-0001-9473-1776

https://orcid.org/0000-0001-7817-747X

\section{REFERENCES}

1. Treasure J, Zipfel S, Micali N, Wade T, Stice E, Claudino A, et al. Anorexia nervosa. Nat Rev Dis Primers 2015;1:15074.

2. Smink FRE, van Hoeken D, Oldehinkel AJ, Hoek HW. Prevalence and severity of DSM-5 eating disorders in a community cohort of adolescents. Int J Eat Disord 2014;47:610-619.

3. American Psychiatric Association. DSM 5. Diagnostic and Statistical Manual of Mental Disorders. 5th Ed. Madrid: Editorial Médica Panamericana; 2014.

4. De Young KP, Lavender JM, Steffen K, Wonderlich SA, Engel SG, Mitchell JE, et al. Restrictive eating behaviors are a nonweight-based marker of severity in anorexia nervosa. Int J Eat Disord 2013;46:849-854.

5. Hale MD, Logomarsino JV. The use of enteral nutrition in the treatment of eating disorders: a systematic review. Eat Weight Disord 2019;24:179198.

6. Westmoreland P, Krantz MJ, Mehler PS. Medical complications of anorexia nervosa and bulimia. Am J Med 2016;129:30-37.

7. Hay PJ, Touyz S, Claudino AM, Lujic S, Smith CA, Madden S. Inpatient versus outpatient care, partial hospitalisation and waiting list for people with eating disorders. Cochrane Database Syst Rev 2019;1:CD010827.

8. Redgrave GW, Coughlin JW, Schreyer CC, Martin LM, Leonpacher AK, Seide M, et al. Refeeding and weight restoration outcomes in anorexia nervosa: Challenging current guidelines. Int J Eat Disord 2015;48:866873.

9. Murray SB, Quintana DS, Loeb KL, Griffiths S, Le Grange D. Treatment outcomes for anorexia nervosa: a systematic review and meta-analysis of randomized controlled trials. Psychol Med 2019;49:535-544.

10. Iniesta Sepúlveda M, Nadeau JM, Whelan MK, Oiler CM, Ramos A, Riemann BC, et al. Intensive family exposure-based cognitive-behavioral treatment for adolescents with anorexia nervosa. Psicothema 2017; 29:433-439.

11. Hatch A, Madden S, Kohn MR, Clarke S, Touyz S, Gordon E, et al. In first presentation adolescent anorexia nervosa, do cognitive markers of underweight status change with weight gain following a refeeding intervention? Int J Eat Disord 2010;43:295-306.

12. Fennig S, Brunstein Klomek A, Shahar B, Sarel-Michnik Z, Hadas A. Inpatient treatment has no impact on the core thoughts and perceptions in adolescents with anorexia nervosa. Early Interv Psychiatry 2017;11: 200-207.

13. Hagman J, Gardner RM, Brown DL, Gralla J, Fier JM, Frank GKW. Body size overestimation and its association with body mass index, body dissatisfaction, and drive for thinness in anorexia nervosa. Eat Weight Disord 2015;20:449-455.

14. Bardone-Cone AM, Higgins MK, St George SM, Rosenzweig I, Schae- fer LM, Fitzsimmons-Craft EE, et al. Behavioral and psychological aspects of exercise across stages of eating disorder recovery. Eat Disord 2016;24:424-439.

15. Kostrzewa E, van Elburg AA, Sanders N, Sternheim L, Adan RA, Kas MJ. Longitudinal changes in the physical activity of adolescents with anorexia nervosa and their influence on body composition and leptin serum levels after recovery. PLoS One 2013;8:e78251.

16. Allison KC, Spaeth A, Hopkins CM. Sleep and eating disorders. Curr Psychiatry Rep 2016;18:92.

17. Kim KR, Jung YC, Shin MY, Namkoong K, Kim JK, Lee JH. Sleep disturbance in women with eating disorder: Prevalence and clinical characteristics. Psychiatry Res 2010;176:88-90.

18. Pieters G, Theys P, Vandereycken W, Leroy B, Peuskens J. Sleep variables in anorexia nervosa: evolution with weight restoration. Int $\mathrm{J}$ Eat Disord 2004;35:342-347.

19. de Souza L, Benedito-Silva AA, Pires MLN, Poyares D, Tufik S, Calil HM. Further validation of actigraphy for sleep studies. Sleep 2003;26: 81-85.

20. de Mateo Silleras B, Redondo del Río P, Camina Martín A, Soto Célix M, Alonso Torre SR, Miján de la Torre A. [Effect of refeeding on the body composition of females with restrictive anorexia nervosa; anthropometry versus bioelectrical impedance]. Nutr Hosp 2013;28:1717-1724.

21. Kriemler S, Puder J, Zahner L, Roth R, Braun-Fahrländer C, Bedogni G. Cross-validation of bioelectrical impedance analysis for the assessment of body composition in a representative sample of 6- to 13-yearold children. Eur J Clin Nutr 2009;63:619-626.

22. Garner D. Eating Disorder Inventory-3: Professional Manual. Lutz, FL: Psychological Assessment Resources, Inc; 2004.

23. Thompson MA, Gray JJ. Development and validation of a new bodyimage assessment scale. J Pers Assess 1995;64:258-269.

24. Wertheim EH, Paxton SJ, Tilgner L. Test-retest reliability and construct validity of Contour Drawing Rating Scale scores in a sample of early adolescent girls. Body Image 2004;1:199-205.

25. Dion J, Blackburn ME, Auclair J, Laberge L, Veillette S, Gaudreault M, et al. Development and aetiology of body dissatisfaction in adolescent boys and girls. Int J Adolesc Youth 2015;20:151-166.

26. Duchesne AP, Dion J, Lalande D, Bégin C, Émond C, Lalande G, et al. Body dissatisfaction and psychological distress in adolescents: Is selfesteem a mediator? J Health Psychol 2017;22:1563-1569.

27. Caspi A, Amiaz R, Davidson N, Czerniak E, Gur E, Kiryati N, et al. Computerized assessment of body image in anorexia nervosa and bulimia nervosa: comparison with standardized body image assessment tool. Arch Womens Ment Health 2017;20:139-147.

28. Elbelt U, Haas V, Hofmann T, Stengel A, Berger H, Jeran S, et al. Evaluation of a portable armband device to assess resting energy expenditure in patients with anorexia nervosa. Nutr Clin Pract 2015;31:362-367.

29. Hopkins WG, Marshall SW, Batterham AM, Hanin J. Progressive statistics for studies in sports medicine and exercise science. Med Sci Sports Exerc 2009;41:3-13.

30. Hopkins WG. A spreadsheet to compare means of two groups. Sportscience 2007;11:22-24.

31. El Ghoch M, Calugi S, Bernabè J, Pellegrini M, Milanese C, Chignola E, et al. Sleep patterns before and after weight restoration in females with anorexia nervosa: a longitudinal controlled study. Eur Eat Disord Rev 2016;24:425-429.

32. Lehmann CS, Hofmann T, Elbelt U, Rose M, Correll CU, Stengel A, et al. The role of objectively measured, altered physical activity patterns for body mass index change during inpatient treatment in female patients with anorexia nervosa. J Clin Med 2018;7. pii: E289.

33. Haas V, Kent D, Kohn MR, Madden S, Clarke S, Briody J, et al. Incomplete total body protein recovery in adolescent patients with anorexia nervosa. Am J Clin Nutr 2018;107:303-312.

34. Agüera Z, Romero X, Arcelus J, Sánchez I, Riesco N, Jiménez-Murcia $\mathrm{S}$, et al. Changes in body composition in anorexia nervosa: predictors of recovery and treatment outcome. PLoS One 2015;10:e0143012. 
35. Mika C, Herpertz-Dahlmann B, Heer M, Holtkamp K. Improvement of nutritional status as assessed by multifrequency BIA during 15 weeks of refeeding in adolescent girls with anorexia nervosa. J Nutr 2004;134: 3026-3030.

36. Tubić B, Pettersson C, Svedlund A, Forslund HB, Magnusson P, Swolin-Eide D. Increased bone mineral content during rapid weight gain therapy in anorexia nervosa. Horm Metab Res 2016;48:664-672.

37. Hillen S, Dempfle A, Seitz J, Herpertz-Dahlmann B, Bühren K. Motivation to change and perceptions of the admission process with respect to outcome in adolescent anorexia nervosa. BMC Psychiatry 2015;15: 140 .

38. Kodama N, Moriguchi Y, Takeda A, Maeda M, Ando T, Kikuchi H, et al. Neural correlates of body comparison and weight estimation in weight-recovered anorexia nervosa: a functional magnetic resonance imaging study. Biopsychosoc Med 2018;12:15.

39. Sala L, Mirabel-Sarron C, Pham-Scottez A, Blanchet A, Rouillon F, Gorwood P. Body dissatisfaction is improved but the ideal silhouette is unchanged during weight recovery in anorexia nervosa female inpatients. Eat Weight Disord 2012;17:e109-e115.

40. Bamford BH, Attoe C, Mountford VA, Morgan JF, Sly R. Body checking and avoidance in low weight and weight restored individuals with anorexia nervosa and non-clinical females. Eat Behav 2014;15:5-8.

41. Marzola E, Nasser JA, Hashim SA, Shih PA, Kaye WH. Nutritional rehabilitation in anorexia nervosa: review of the literature and implications for treatment. BMC Psychiatry 2013;13:290.

42. Gianini LM, Klein DA, Call C, Walsh BT, Wang Y, Wu P, et al. Physical activity and post-treatment weight trajectory in anorexia nervosa. Int $\mathrm{J}$ Eat Disord 2016;49:482-489. 


\begin{tabular}{|c|c|}
\hline Descriptor & Treatment Program for Eating Disorders \\
\hline In operation since & 2001 \\
\hline Days a week & 5 (Monday to Friday; from 9 am to $21 \mathrm{pm}$ ) \\
\hline Duration of treatment & 18-20 weeks generally (depending on the individual evolution of each patient) \\
\hline Treatment orientations & Cognitive behavior \\
\hline Group/individual treatment & Mostly group (individual sessions in parallel with the psychiatrist and the staff in charge) \\
\hline Group size & $8-10$ \\
\hline Group structure & Open \\
\hline Behavioral contract & Yes (patients and legal guardians) \\
\hline Inclusion criteria & $\begin{array}{l}\text { DSM-5 eating disorder: AN, BN and EDNOS } \\
\text { Patients between } 0 \text { and } 16 \text { years old } \\
\text { State of severe malnutrition and/or somatic complications } \\
\text { Negative attitude toward the ingestion of food } \\
\text { Failure in outpatient treatment } \\
\text { High probability of failure due to the degree of chronicity } \\
\text { Excessive and uncontrolled physical exercise }\end{array}$ \\
\hline Exclusion criteria & $\begin{array}{l}\text { Acute medical risk that requires total hospitalization } \\
\text { Acute risk of suicide and/or very serious psychopathology that requires total hospitalization } \\
\text { Serious abuse of substances/toxins that clearly interfere with the normalization of weight, appetite and meal }\end{array}$ \\
\hline Goals of treatment & $\begin{array}{l}\text { Normalization of weight or weight gain (nutritional rehabilitation through adequate caloric intake) } \\
\text { Reeducation and normalization of eating behavior } \\
\text { Identification and resolution of perpetuating factors } \\
\text { Control of constants and analytics according to patient's evolution } \\
\text { Restoration and stabilization of healthy eating patterns (e.g., promoting a more social meal) } \\
\text { Improvement of aspects associated with ED (emotional regulation, self-esteem, interpersonal relationships, motivation, etc.) } \\
\text { Identification of the underlying pathological processes, both psychological and family relationships. }\end{array}$ \\
\hline Weight control & Individual weight measurement twice a week (Monday and Friday) \\
\hline Eating and compensation behavior & $\begin{array}{l}\text { Meal plan } \\
\text { Supervised meals } \\
\text { Self-monitoring (food diary) } \\
\text { Supervised cooking } \\
\text { Psychoeducation }\end{array}$ \\
\hline Body attitude & $\begin{array}{l}\text { Body awareness group } \\
\text { Positive reinforcement } \\
\text { Clothing control } \\
\text { Relaxation training }\end{array}$ \\
\hline Coping skills & $\begin{array}{l}\text { Social skills training } \\
\text { Health education } \\
\text { Food education } \\
\text { Leisure activities weekly: visits to the beach, cinema, factories, etc. }\end{array}$ \\
\hline Interpersonal functioning & Group psychotherapy \\
\hline Nonverbal expression & $\begin{array}{l}\text { Art therapy } \\
\text { Music therapy }\end{array}$ \\
\hline Family functioning & Family therapy \\
\hline Biology & Medication/medical monitoring weekly \\
\hline Treatment planning & Re-evaluation of treatment plan weekly \\
\hline Other & School follow-up and motivational group \\
\hline
\end{tabular}

Based on the descriptors proposed by Lammers et al. ${ }^{1}$ to compare different DHPs for TPED

\section{REFERENCE}

1. Lammers MW, Exterkate CC, De Jong CA. A Dutch day treatment program for anorexia and bulimia nervosa in comparison with internationally described programs. Eur Eat Disord Rev 2007;15:98-111. 